\title{
A Study on the Compliance, Risk Perception and Attitude of University of Khartoum Medical Students to Safety Measures Against COVID-19 during the Period of Lockdown, Khartoum, Sudan
}

Mustafa Mohamed Ibrahim Ali

University of Khartoum Faculty of Medicine

Khabab Abbasher Hussien Mohamed Ahmed

University of Khartoum, Faculty of Medicine

Mohammed Eltahier Abdalla Omer ( $\square$ Mohammedeltahier100@gmail.com )

MBBS, Gadarif University, Faculty of Medicine and Health Sciences

\section{Research Article}

Keywords: COVID-19, SARS-CoV-2, adherence, risk perception, medical students

Posted Date: June 25th, 2021

DOl: https://doi.org/10.21203/rs.3.rs-658797/v1

License: (c) (i) This work is licensed under a Creative Commons Attribution 4.0 International License.

Read Full License 


\section{Abstract \\ Background}

COVID-19 is a novel respiratory infectious disease. Preventive measures against COVID-19 include facemask use, physical distancing and good sanitation and hygiene practices. Risk perception of COVID19 is important in prevention. Lockdown was imposed in Sudan in early 2020.

\section{The study aims to:}

study the compliance, risk perception and attitude of University of Khartoum Medical Students to safety measures during the period of COVID-19 lockdown.

\section{Methodology:}

An observational descriptive cross sectional study, institutional based at the University of Khartoum, Faculty of Medicine in 435 students selected via proportionate stratified random sampling through a selfadministered semi- structured questionnaire. Data was analyzed using SPSS version 20. Pearson and Mann Whitney $U$ test were used to measure associations.

\section{Result}

Out of 435 participants, a mean adherence of $57.7 \%$, mean risk perception of $73.6 \%$ were elicited. And $80.3 \%$ had a positive attitude towards lockdown. Adherence had significant association with gender and risk perception. Risk perception had no significant relation with gender.

\section{Conclusion}

In conclusion, adherence to preventive measures among medical students of the University of Khartoum was poor. Risk Perception was high. Attitude towards lockdown was positive.

\section{Background}

Coronavirus disease 2019(1)(COVID-19) also known as 2019-nCoV(2)[a novel severe acute respiratory syndrome (SARS) (1) was declared a pandemic by the World Health Organization (WHO) on March 11, 2020. The novel virus first appeared in Wuhan City, in the Hubei Province of China at the end of December, 2019(2-5). The virus continued to spread around globally with people from many countries suffering the death of loved ones and difficult financial situations(5). The clinical disease termed COVID19 is caused by a new betacoronavirus, now given the name SARS- CoV-2. SARSCoV2 shares 79\% 
sequence identity with SARS CoV. SARS CoV was the virus that caused the major SARS outbreak in 20022003(4). Transmission of the virus is mainly through respiratory droplets or via contact with infected secretions. Spread via contaminated surfaces is also probable(4).

Symptoms include fever, fatigue, cough, and difficulty breathing and ranged from mild to severe, even leading to respiratory failure and death. Symptoms were seen to appear in people 1 week following exposure to the virus(5) At the end of January 2020, it was confirmed that this infection can be spread from person to person, especially through close contact with an infected person within 14 days of the onset of symptoms. In addition, it has been established that infection can transfer from a person carrying the virus without demonstrating signs or symptoms of infection (asymptomatic).(5)

Preventive measures, which include observing proper hand hygiene(5) through increased hand washing and use of sanitizing hand gels, covering coughs(6), maintaining social distancing, avoiding crowded places, $(5)$ wearing masks $(6,7)$ and observing quarantine protocols. Furthermore, closing public places such as schools, restaurants, barbershops, and workplaces whilst letting people work remotely from home have also been introduced to reduce the spread of the virus(5). The countermeasures include a wide range of behaviours. In some cases, the public may request temperature monitoring (for example, at an airport). Sometimes home isolation or home isolation is recommended. (6) The psychosocial factors that influence the previous comments include perceived vulnerability (perceived risk), perceived severity, perceived benefits, and perceived barriers. (6) In early 2020, countries rely on non-pharmaceutical interventions (NIP) to respond to the COVID19 epidemic. In non-profit organizations, quarantine interventions have proven to be very effective in reducing the spread of COVID19 (8). And restrict the movement of people who may be exposed to infectious diseases.

. The primary purpose of quarantine is to prevent transmission of an infectious agent from those potentially incubating it.(9) Self-quarantine is not the same as isolation, which separates people known to be ill from others who are not. (1)

Governments around the world are working hard to contain the spread of COVID19. A major component of these efforts applied by public health authorities is self-quarantine. $(1,10)$

COVID-19 infection is confirmed to be able to transmit from person to person, especially when there has been close contact with infected individuals within 14 days of the onset of symptoms $(5,11)$. To limit the spread of COVID-19 infection, the strategy being adopted is to encourage people to follow preventive measures, which include observing proper hand hygiene, maintaining social distancing, avoiding crowded places, and observing quarantine protocols. Furthermore, closing public places such as schools, restaurants, barbershops, and workplaces whilst letting people work remotely from home have also been introduced to reduce the spread of the virus $(5,12)$. Therefore it is important to assess the compliance rate of the population to these measures to make sure of the efficiency of these measures and try to identify the obstacles and difficulties associated with following these measures and address these problems in order to relieve or solve them. 


\section{Objectives:-}

\section{General Objective:}

To study the Compliance, risk perception and attitude of University of Khartoum

Medical Students to Safety measures During the Period of COVID-19 lockdown.

\section{Specific Objectives:}

1.To determine the practices of the following among University of Khartoum medical students during the period of COVID-19 lockdown.
a. Facemask use.
b. Social Distancing.
c. Sanitation and hygiene.

2. To identify factors associated with difficulty of compliance with safety measures faced by University of Khartoum medical students during the period of COVID-19 lockdown.

3. To demonstrate risk perception of University of Khartoum medical students towards coronavirus disease 2019 (COVID-19).

To determine the attitude of University of Khartoum medical students towards lockdown measures.

\section{Material And Method}

Study design:

An observational descriptive cross sectional study, institutional based.

Study Area:

The University of Khartoum, Faculty of medicine. Established in 1924 by Lord Kitchener. Initially called Gordon's Memorial College, the university was founded in 1902 by the British colonizer. The faculty of medicine was founded by Lord Kitchener in 1924 and then both institutes were joined together under the name "The University of Khartoum" which was the first university in Sudan and one of the first universities in Africa. It is home to the top students in Sudan and some of the most brilliant minds to have ever passed the country. It was nicknamed "The beautiful impossible" by its students and the public 
as it was nearly impossible to get into as due to its society and scenery. The faculty of medicine of the university lies in Alqasr Ave. in Khartoum City in Khartoum State in Sudan.

Study Population:

Medical students registered at the University of Khartoum. From first year to sixth year. Seven batches in total as there are currently seven batches registered at the faculty of medicine. Total number of students is 2341 .

Inclusion Criteria:

1. Undergraduate student registered at the faculty of medicine, University of Khartoum.

Exclusion Criteria:

1. Students who refuse to participate.

2. Students who were outside Sudan during lockdown.

\section{Sampling:-}

Sample Size:

By using the equation: $n=N /\left(1+N d^{\wedge} 2\right) n=$ Sample size.

$\mathrm{N}=$ Size of the population $=2341$

$d=$ tolerated margin of error, $5 \%(0.05)$ for a $95 \%$ confidence interval.

$\mathrm{n}=2341 /(1+2341 \times .05 \times .05)=2341 /(1+5.8525)=2341 / 6.8525=341$ samples.

Number of samples from each batch $=$ (number of students in the batch/total

number of medical students) $\times 341$

Sampling Technique:

Proportionate stratified random sampling technique was used.

\section{Data Collection:-}

Web based semi-structured questionnaire was distributed to samples by means of Google Form.

\section{Data Analysis:-}

Entry: 
Google spreadsheet was downloaded, converted into excel sheet and inserted into SPSS, then it was coded.

Analysis:

Analysis was done using SPSS version 20. Data is presented as frequencies and percentages using figures and tables. Pearson correlation test was used to test

correlation between overall adherence and risk perception.

Spearman test was used to test correlation between each type of adherence.

A ( $P$ value less than 0.05$)$ is considered significant.

Ethical Consideration:

This research was conducted after obtaining ethical approval from the Community Medicine department at the Faculty of Medicine at the University of Khartoum.

Permission:

Written consent was obtained from all participants.

Limitations of the study:

The study was conducted in one faculty from one university, therefore the results of this study cannot be generalized to include medical students from other schools of medicine and other universities. Secondly, the questionnaire was a self- administered questionnaire in the form of google form and was not interview based.

\section{Results}

Demographic characteristics and source of knowledge about safety measures:

In this study, 435 participants were enrolled. The majority of them was female $(60 \%)$. The majority was also single (99.8\%). The majority resided with family during lockdown (85\%). Most were inside Khartoum (84\%). Monthly income of most participants is " 15000 SDG or above" (45\%). The mean age was 21 (SD = 2.1). The most common source of knowledge about safety measures against COVID-19 was "social media" followed by "TV" then "family members and friends". (Table 1). 
Table 1

Demographic characteristics

\begin{tabular}{|c|c|c|}
\hline $\begin{array}{l}\text { Demographic } \\
\text { characteristics }\end{array}$ & & Frequencies (\%) \\
\hline \multirow[t]{2}{*}{ gender } & Female & $261(60 \%)$ \\
\hline & Male & $174(40 \%)$ \\
\hline \multirow[t]{2}{*}{ Marital status } & Married & $1(0.2 \%)$ \\
\hline & Single & 434(99.8) \\
\hline \multirow[t]{2}{*}{ Residence site } & Inside Khartoum & $366(84 \%)$ \\
\hline & Outside Khartoum & $69(16 \%)$ \\
\hline \multirow[t]{6}{*}{ Residence with } & Alone & $2(0.5 \%)$ \\
\hline & Friend & $1(0.2 \%)$ \\
\hline & In dorms & $41(9.4 \%)$ \\
\hline & Outside Sudan & $1(0.2 \%)$ \\
\hline & With family & $370(85.1 \%)$ \\
\hline & With Relatives & $20(4.6 \%)$ \\
\hline \multirow[t]{4}{*}{ Monthly income } & less than 5000 SDG & $72(16.6 \%)$ \\
\hline & $5000-10,000$ SDG & $101(23.2 \%)$ \\
\hline & $10,000-15,000$ SDG & $68(15.6 \%)$ \\
\hline & 15,000 SDG and more & $194(44.6 \%)$ \\
\hline \multirow[t]{2}{*}{ grade } & 6 th grade & $49(11.3 \%)$ \\
\hline & 5 th grade & $62(14.3 \%)$ \\
\hline
\end{tabular}

\begin{tabular}{|ll|}
\hline 4th grade & $\mathbf{5 5 ( 1 2 . 6 \% )}$ \\
\hline 3rd grade & $68(15.6 \%)$ \\
\hline 2nd grade & $65(14.9 \%)$ \\
\hline 1st grade semester two & $60(13.8 \%)$ \\
\hline 1st grade semester one & $76(17.5 \%)$ \\
\hline Total = 435 & \\
\hline
\end{tabular}

Facemask use Practice: 
Out of all participants, $2 \%$ believed that facemasks are not effective in preventing the transmission of the covid-19 virus and $10 \%$ did not use facemasks during lockdown. Type of mask used most was disposable masks (58\%) followed by fabric masks (32\%) .Out of all participants, $11 \%$ lowered their mask to eat, drink or smoke in public, $47 \%$ lowered it when talking to close friends or relatives. Around $46 \%$ did not wear facemasks when visiting relatives or friends. A majority of $61 \%$ did not wear facemasks in the car, $13 \%$ did not wear it at the supermarket and $7 \%$ did not wear it at hospitals and clinics.

The main obstacle to using facemasks was that facemasks caused the feeling of being suffocated (reported by 213 participants) (about 49\%). The second most common obstacle faced was that facemasks cause skin problems (156 responses) (around 36\%).

There is no significant relation between adherence to social/ physical distancing practices and belief in their role in prevention of transmission of COVID-19.

Risk Perception:

Almost $66.7 \%$ of participants gave a maximum score of " 5 " when asked how worried they were about their loved ones becoming infected with COVID-19, making it the commonest answer recorded.

While $32.4 \%$ of participants gave a score of " 5 " regarding how prevalent they thought coronavirus disease was in Sudan, 31.3\% gave a score of " 4 ", making these two scores the most common answers recorded.

Around $41.2 \%$ of participants answered with a score of " 3 " regarding how deadly they thought coronavirus disease was, making it the most common response recorded.

Around $34.5 \%$ of participants answered with a score of " 5 " on how probable they thought it was for them to become infected with COVID-19 if they did not apply preventive measures, marking the commonest recorded response.

The commonest response given to how worried the participants were about becoming infected with COVID-19 was the answer " 3 " on the Likert scale. Given by $28.5 \%$ of participants.

Attitude towards lockdown:

A majority of participants (68.3\%) stated they complied with lockdown measures because they were afraid their loved ones would become infected with COVID-19 making it the commonest option checked on the checklist. Around $7.6 \%$ of participants stated they complied because other people were complying. Around $7 \%$ of participants stated that they did not comply with lockdown measures.

\section{Discussion}


This is the first paper on the topic of risk perception, adherence to covid-19 preventive measures and attitude towards lockdown in medical students of Sudan. The main source of knowledge about COVID-19 preventive measures was found to be social media followed by TV then family and friends. This is consistent with a theory made by another study which stated that although it did not investigate the source of knowledge it is likely that it was mass media.(3) This may be due to the frequent use of mobile devices by people in this age group as well as watching more television as people had to spend more time at home.

In this study, the overall response rate was $127 \%$. The average overall adherence to preventive measures score was 15 (SD = 3.7) out of 26 giving a percentage of $57.69 \%$ which is markedly less than results of a similar study in Iran which evaluated adherence of medical students that found the average adherence level to be $94.47 \%(16)$ This contradiction may be due to differences in socio- economic situations between participants in this study and participants in the study conducted in Iran. Perhaps, level knowledge about COVID-19 and preventive measures played a role in adherence.

Overall mean adherence to preventive measures among females was found to be higher than that among males as the average score among females was 15.3 and that of males was 14.4. Mann Whitney $U$ test was significant with a ( $\mathrm{p}$ value of 0.06 ). This is similar to a study conducted in Egypt by Enayat M. Soltan et Al. which found that females have higher percentages in regard to practicing preventive behaviors $(P<$ 0.001).(13) This could be due to that females are more observant of cleanliness and how society views them and this reflects on the cultural background of the Sudanese community.

There was no significant relation between adherence of medical students of the University of Khartoum to preventive measures and whether they resided inside or outside Khartoum during lockdown. This could be due to their knowledge about COVID-19 as well as communicable diseases in general and the preventive measures associated with them.

Regarding adherence to facemask use, $90 \%$ of participants used facemasks during lockdown. This is significantly higher than the $50 \%$ figure reported by a similar study conducted on the Somali community by Mohammed A. M. Ahmed et Al.(14) However, only $6.4 \%$ used them correctly. This could be related to the high degree of risk perception towards COVID-19 recorded from participants as the majority were worried about their loved ones becoming infected, and to a lesser degree themselves becoming infected. The study found that $80 \%$ of participants reported that they wore facemasks on the street which is significantly higher than the $45.5 \%$ figure reported by a similar study conducted in Brazil by Edlaine Faria de Moura Villela et Al.(15) Which was a community based study. This could be due to the efforts of campaigns raising knowledge and advocating application of preventive measures as well as the high degree of risk perception recorded among participants.

Questions about social distancing were in the form of Likert-scale statements. 5 options were given starting from "Never" followed by "Rarely" then "Sometimes", "Usually" and "Always". 31\% of participants (around one third) said they never attended social gatherings which is a significantly lower percentage than a study conducted in Saudia Arabia by Nouf Alotaibi et Al. (5) which showed that around 75\% of 
participants would never attend social gagathering (5) This could be due to scarcity of entertainment and activities during lockdown as well as the cultural background of the Sudanese community. $31 \%$ of participants stated they never kept safe distance from others which is significantly higher than the Saudi study which reported $4 \%$ would never keep safe distance. This could be due to the high level of boredom recorded from participants.

Regarding sanitation and hygiene practices $98 \%$ of participants believed it had a role in preventing virus transmission similar to a study conducted by Enayat M. Soltan et Al. which found that $92.2 \%$ know the importance of handwashing and hygiene(15) This could be directly related to knowledge of medical students regarding communicable diseases. $93 \%$ of the participants in this study reported that they performed acts of sanitation and hygiene during lockdown. The majority of participants (52\%) washed their hands for 10-20 seconds which is less time than that recommended by the WHO and the CDC in their guidelines.(17) There was a significant relation between facemask use adherence and belief of facemask effectiveness in prevention of COVID-19 transmission.

There was a significant relation between keeping good sanitation and hygiene practices and belief of their role in prevention of the transmission of the virus. This could very well be related to the knowledge of medical students about communicable diseases and infectious agents.

There was weak positive correlation between adherence to one safety measure and other safety measure possibly indicating that whichever source of knowledge a participant drew their knowledge from, it didn't leave out an aspect of preventive measures. Meaning it is probable that participants' perception of each aspect of preventive measures is relatively close to another.

Factors associated with difficulty of adherence were presented in the form of a checklist adding an option for the participant to write an option if it was not presented in the questionnaire. The main obstacle for facemask use was found to be that facemasks cause the feeling of suffocation reported by 213 participants (48\%) followed by skin problem causation, communication hindrance and fogging of glasses, $156,137,133$ responses respectively in close proximity of each other.

This could be due to that medical students had to wear facemasks for extended periods of time.

The major obstacles to social (physical) distancing were found to be boredom as 206 participants checked it on the checklist (47.3\%) and non-application of safety measures by other people at 201 responses (46.2\%). Anxiety was third in line at 133 recorded responses (30\%).

The major obstacles to keeping good sanitation and hygiene practices was that respondents found it tiring to keep washing their hands at most times recorded by 194 respondents (44.6\%). 155 participants $(35.6 \%)$ recorded that hand sanitizers were unavailable for purchase during lockdown. 139 participants (32\%) recorded that prices of hand sanitizers were expensive.

Risk perception was assessed based on 5 Likert-scale questions and the average score was 18.4 (SD = 3.7) which is equivalent to $73.6 \%$ which is significantly higher than a study conducted in China by Yaping 
Zhong et Al. which reported an average risk perception of $42 \%$ among Chinese COVID-19 patients in one of the Fang Cang hospitals in Wuhan city, China in February 2020.(18). This could be due to that patients were already diagnosed with COVID-19 and therefore they wouldn't be afraid of acquiring it. However, the high risk perception recorded among medical students could be related to their age mean $=21$ as at this point they would be getting ready to accomplish their hopes and dreams.

The majority of participants showed great worry about their loved ones becoming infected as 290 participants answered with the maximum response grade of " 5 " to how worried they were about their loved ones becoming infected making said response the commonest response to the question. On the question regarding probability of becoming infected if one didn't follow preventive measures the commonest answer was also the maximum response grade of " 5 " with 150 responses recorded. From these results, respondents were more worried about their loved ones becoming infected than themselves, similar to the study conducted in Brazil by Edlaine Faria de Moura Villela et Al.(15) This could be related to the nature of human beings as well as the cultural and religious background of the Sudanese community.

$84 \%$ of participants answered with a grade of 3 or above to how deadly they perceive COVID-19 as. Hence, the majority of participants recognized COVID-19 as a life threatening disease which is similar to what Enayat M. Soltan et Al. found in her study as $83 \%$ of participants in their study thought COVID-19 is a life-threatening illness.(15) This could be due to knowledge about COVID-19 but could also be related to rumors in society.

Pearson correlation found that there is a significant positive medium strength correlation between level of adherence and risk perception $(p<0.001 r=0.32)$ which is in contrast with a study conducted in Iranian medical students by Mohammad Hossein Taghrir et Al. which found a negative significant correlation between self-reported preventive behaviors and risk perception.(16) However, this result is similar to that found by M. Guillon et Al. in their study.(8) This could be due to the level of strictness of authorities in imposing lockdown.

There was no significant relation between risk perception and gender $(p$ value $=0.8)$ and the median risk perception of females was found to be 20 while that of males was found to be 18 slightly higher in females. This is in contrast to a study in Iran which reported that there was a significant difference between males and females in risk perception, with risk perception being lower among females.(16) This could be related to the difference in cultural backgrounds between Sudan and Iran.

The attitude of medical students who participated in this study towards lockdown was assessed based on three items. $80.3 \%$ of participants agreed with the imposition of lockdown as a preventive measure while $19.7 \%$ disagreed. $58.5 \%$ thought benefits from lockdown outweighed its disadvantages showing that over half of the sample had a positive attitude towards lockdown. This could be related to background views on public health importance. As it may be related to risk perception, as from results, participants were highly worried about their loved ones and themselves. However, the marked difference between the $80.3 \%$ and $58.5 \%$ figures could be due to socio-economic situations. This is similar to the 
results of a study conducted in France by Patrick Peretti-Watel et Al. which found that most participants supported lockdown as the only effective way to fight the epidemic and the need to maintain it for several more weeks; however, this support was significantly lower among low income respondents.(18)

\section{Conclusion}

In conclusion, adherence to preventive measures practice among medical students of the University of Khartoum during lockdown was poor. Risk Perception of COVID-19 was high. Attitude towards lockdown was positive in over half of participants. Major obstacles to compliance were determined and were found to be sensation of suffocation by facemasks, boredom for social/physical distancing and being weary of washing hands with water and soap frequently.

\section{Recommendation:}

Based on findings, it is highly important to improve pre-existing campaigns as well as start new ones to raise awareness on COVID-19 and preventive measures against it as there were many participants who reported that they adhered to safety measures but were fund to be incorrectly doing so. It's also important to better the quality of teaching methods and curricula to try to ensure medical students would have sufficient knowledge as well as positive attitude in case of future epidemics or pandemics. It's also highly important to try and overcome factors associated with difficulty of adherence to preventive measures against infectious diseases.

\section{Declarations}

\section{Consent for publication}

Not applicable.

\section{Availability of data and materials}

The materials datasets used and/or analyzed during this study are available from the corresponding author on reasonable request.

\section{Competing interests}

The authors declare that they have no competing interests.

\section{Funding}

This research did not receive any specific grant from funding agencies in the public, commercial, or notfor-profit sectors.

\section{Authors' contributions}


All authors participated in planning the study, data collection, results and discussion sections.

\section{References}

1. Bodas M, Peleg K. Self-isolation compliance in the COVID-19 era influenced by compensation: Findings from a recent survey in Israel. Health Aff. 2020;39(6):936-41.

2. Roma P, Monaro M, Muzi L, Colasanti M, Ricci E, Biondi S, et al. How to improve compliance with protective health measures during the covid-19 outbreak: Testing a moderated mediation model and machine learning algorithms. Int J Environ Res Public Health. 2020;17(19):1-17.

3. Gallè F, Sabella EA, Da Molin G, De Giglio O, Caggiano G, Di Onofrio V, et al. Understanding knowledge and behaviors related to covid - 19 epidemic in italian undergraduate students: The epico study. Int J Environ Res Public Health. 2020;17(10):1-11.

4. Lake MA. What we know so far: COVID-19 current clinical knowledge and research. Clin Med J R Coll Physicians London. 2020;20(2):124-7.

5. Alotaibi N, Almutairi S, Alotaibi M, Alotaibi MM, Alsufian T. The Extent of Commitment of Saudis During Holy Ramadan to Social Distancing Measures Required for the Prevention of Transmission of COVID-19. J Community Health [Internet]. 2020;(0123456789). Available from: https://doi.org/10.1007/s10900-020-00936-3

6. Gershon RR, Zhi Q, Chin AF, Nwankwo EM, Gargano LM. Adherence to Emergency Public Health Measures for Bioevents: Review of US Studies. Disaster Med Public Health Prep. 2018;12(4):528-35.

7. Cava MA, Fay KE, Beanlands HJ, McCay EA, Wignall R. Risk perception and compliance with quarantine during the SARS outbreak. J Nurs Scholarsh. 2005;37(4):343-7.

8. Guillon M, Kergall P. Attitudes and opinions on quarantine and support for a contact-tracing application in France during the COVID-19 outbreak. Public Health [Internet]. 2020;188:21-31. Available from: https://doi.org/10.1016/j.puhe.2020.08.026

9. Saurabh K, Ranjan S. Compliance and Psychological Impact of Quarantine in Children and Adolescents due to Covid-19 Pandemic. Indian J Pediatr. 2020;87(7):532-6.

10. Reynolds DL, Garay JR, Deamond SL, Moran MK, Gold W, Styra R. Understanding, compliance and psychological impact of the SARS quarantine experience. Epidemiol Infect. 2008;136(7):997-1007.

11. Singhal T. Review on COVID19 disease so far. Indian J Pediatr. 2020;87(April):281-6.

12. Chen HC, Xu W, Paris C, Reeson A, Li X. Social distance and SARS memory: Impact on the public awareness of 2019 novel coronavirus (COVID-19) outbreak. medRxiv. 2020;

13. Faria de Moura Villela E, López RVM, Sato APS, de Oliveira FM, Waldman EA, Van den Bergh R, et al. COVID-19 outbreak in Brazil: adherence to national preventive measures and impact on people's lives, an online survey. BMC Public Health. 2021;21(1):1-10.

14. Ahmed MAM, Fodjo JNS, Gele AA, Farah AA, Osman S, Guled IA, et al. Covid-19 in somalia: Adherence to preventive measures and evolution of the disease burden. Pathogens. 2020;9(9):1-11. 
15. Soltan EM, El-Zoghby SM, Salama HM. Knowledge, Risk Perception, and Preventive Behaviors Related to COVID-19 Pandemic Among Undergraduate Medical Students in Egypt. SN Compr Clin Med. 2020;2(12):2568-75.

16. Taghrir MH, Borazjani R, Shiraly R. COVID-19 and iranian medical students; A survey on their relatedknowledge, preventive behaviors and risk perception. Arch Iran Med [Internet]. 2020;23(4):249-54. Available from: https://doi.org/10.34172/aim.2020.06

17. Mehta N, Qiao R. Medical management of COVID-19 clinic. J Biomed Res [Internet]. 2020;34(6):41621. Available from: https://apps.who.int/iris/handle/10665/332196 